\title{
2D-Tasks for Cognitive Rehabilitation
}

\author{
R. Caballero Hernández ${ }^{1,2}$, J.M. Martínez Moreno ${ }^{1,2}$, A. García Molina ${ }^{3}$, S. Ferrer Celma ${ }^{4}$, J. Solana \\ Sánchez $^{1,2}$, R. Sánchez Carrión ${ }^{3}$, E. Fernández Casado ${ }^{4}$, R. Pérez Rodríguez ${ }^{1,2}$, A. Gómez Pulido ${ }^{3}$, C. \\ Anglès Tafalla ${ }^{4}$, C. Cáceres Taladriz ${ }^{1,2}$, M. Ferré Bergada ${ }^{4}$, T. Roig Rovira ${ }^{3}$, P. García López ${ }^{4}$, J.M. \\ Tormos Muñoz ${ }^{3}$, E.J. Gómez Aguilera ${ }^{1,2}$ \\ ${ }^{1}$ Grupo de Bioingeniería y Telemedicina, Universidad Politécnica de Madrid, Madrid, España \\ ${ }^{2}$ Centro de Investigación Biomédica en Red en Bioingeniería, Biomateriales y Nanomedicina, Madrid, España \\ ${ }^{3}$ Instituto Universitario de Neurorrehabilitación Guttmann-UAB, Barcelona, España \\ ${ }^{4}$ Grupo de Investigación de Arquitecturas y Servicios Telemáticos, Universidad Rovira i Virgili, Tarragona, España
}

\begin{abstract}
Neuropsychological Rehabilitation is a complex clinic process which tries to restore or compensate cognitive and behavioral disorders in people suffering from a central nervous system injury. Information and Communication Technologies (ICTs) in Biomedical Engineering play an essential role in this field, allowing improvement and expansion of present rehabilitation programs.
\end{abstract}

This paper presents a set of cognitive rehabilitation 2D-Tasks for patients with Acquired Brain Injury (ABI). These tasks allow a high degree of personalization and individualization in therapies, based on the opportunities offered by new technologies.

Keywords - Adquired Brain Injury (ABI), Cognitive Rehabilitation, PREVIRNEC.

\section{INTRODUCTION}

Nowadays a high percentage of world population is affected by diseases that cause neurological disabilities; hence there is a great need to develop rehabilitation programs in order to restore, minimize or compensate functional and cognitive changes appeared in those affected people [1].

Brain is the most complex structure of human body and the main nerve center; its different areas are mainly responsible for movement, sensations and perceptions, emotions and behavior, being the responsible for all the higher cognitive functions: attention, memory, language and executive functions. Brain damage may affect one or more of these functions to a greater or lesser extent [2].

Attention and memory are the basis of cognitive processes. The attention function filters external information and activates the brain areas that will properly response to this information, as the first step for the rest of cognitive processes. The memory function stores information, after it has been encoded by the attention [3] [4].

Executive functions include several mental processes designed to efficiently solve complex mental and environmen- tal problems, according to individual and society conditions: behaviour and irrelevant information inhibition, selfregulation of emotion, motivation and alertness, planning, decision making, self-monitoring and self-evaluation [5].

The main objective of cognitive neuropsychological rehabilitation [6] is to increase patients' autonomy and quality of life and to reduce the load, stress and isolation suffered by their family, which becomes an essential element in such patients' treatment. ICTs in Biomedical Engineering provide the resources and technical skills needed to enhance and extend the cognitive rehabilitation process in an economically sustainable way [7].

The combination of neuropsychological rehabilitation, virtual environments and collaborative tele-assistance rehabilitation platforms is on the side of the efficacy of the treatment and maximizes the opportunity of the nervous system to reorganize those functions that will restore higher functionality in relation to Activities of Daily Living (ADL), improving patients' quality of life.

The use of ICTs increases rehabilitation effectiveness and facilitates its personalization and efficiency, increasing not only the number of sessions for each patient but also allowing a higher number of patients to be treated by only one therapist, which involves the upgrading and optimization of care system.

At present, several projects attempt to incorporate the potential of ICTs to neurorehabilitation. In 2002 the EuropaNET project (http://europanet.sema.es) developed a teleassistance platform for remote neurological, psychological, educational and social attention in patients that present cognitive impairment.

The Gerontology Research Group at the University of A Coruña and the Supercomputing Centre of Galicia (Spain) developed, between 2004 and 2007, a project called DISCOGNITIOS (http://lembra.cesga.es), which attempted to identify cognitive telerehabilitation software.

The CANTAB [8] neuropsychological tests were developed more than 21 years ago at the University of Cambridge 
(http://www.camcog.com), to enable detailed translational assessment and evaluation of cognitive function.

Among all the possible neuropsychological rehabilitation approaches, in this paper we will focus on new cognitive rehabilitation tasks, supported on the resources offered by ICTs. These tasks try to help in cognitive impairment treatment and specific training of attention, memory and executive functions. Therefore, taking into account the importance of the neurorehabilitation process and the state of the art, the primary objectives of this research work are:

- To improve current neuropsychological rehabilitation by incorporating ICTs.

- To design and develop 2D cognitive rehabilitation tasks which allow customization and individualization of therapies.

- To provide objective data on the outcomes of therapies, offering the clinicians the opportunity to assess the neurorehabilitation process.

\section{Cognitive RehabiLitation tASKS}

Tasks developed in this work are held in PREVIRNEC [9]; this is a cognitive telerehabilitation platform that allows therapists to plan personalized treatments. Those plans are stored on a server, which sends the parameters of the assigned tasks when the patient, asynchronously, connects to the platform. Tasks run locally and send the results back to the server.

From the design and development point of view, the first step consists of a detailed study of tasks specifications, which are generated by the Institut Guttmann's neuropsychologist team.

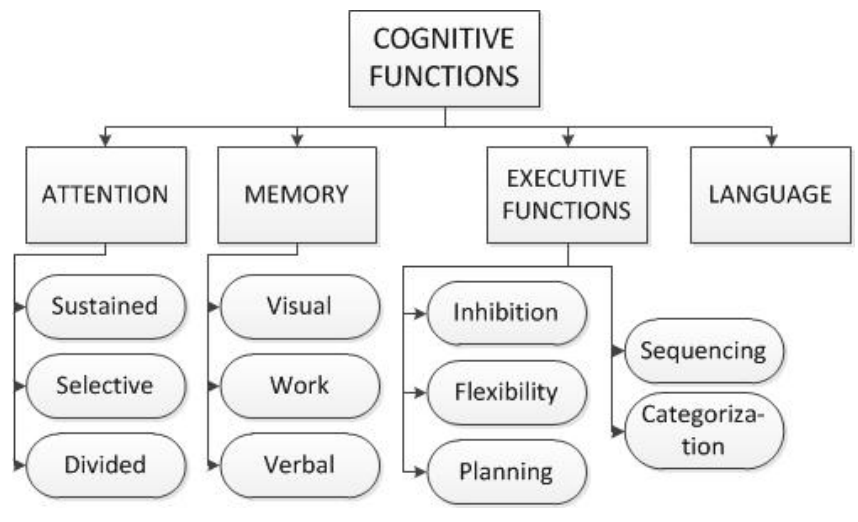

Fig.1 Cognitive Functions and Subfunctions

Each task is carefully focused on the treatment of a specific cognitive function. The tasks presented in this work belong to three of the four higher cognitive functions: atten- tion, memory and executive functions. Each of these categories is subdivided into subcategories, which are specified in Fig. 1.

Task specifications begin with a brief description of the clinical problem (specific cognitive subfunction) that is tried to be rehabilitated. The design and development process of each task is based on the following description.

\section{A. Design}

Every task has a common interface design, which aims to achieve the maximum usability, based on the special characteristics of brain-damaged patients.

In general, a simple and consistent design is desired to make the patient feel familiar with the environment even though the exercises change along the treatment. For improving usability, we have worked on patterns of consistency and coherence in order to maintain uniformity in screen design regarding to their background and the placement of the different elements. Likewise, the same color remains to the common elements of the different tasks for an easier and more intuitive identification.

As for the execution, all the tasks follow a common sequence of well-defined actions. All of them begin with an initial screen, where the instructions to perform the tasks are shown, and end with a final screen where recorded variables, which are previously defined by neuropsychologists, are numerically and graphically displayed in order to summarize the patient's results in the exercise.

Tasks behaviour can be modeled by a state diagram in which each state represents a screen. The transition between states is determined automatically or by a user event, such a mouse click. The detailed sequence between the different states is shown in Fig. 2.

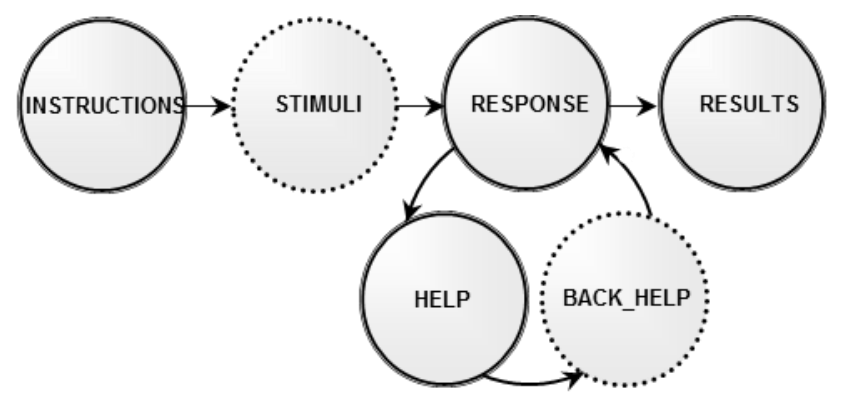

Fig. 2 State Diagram of a Task

\section{- INSTRUCTIONS}

It shows task instructions.

\section{- $\quad$ STIMULI}

This generates task stimuli (images, words, sounds, relationships, etc.). 
- RESPONSE

Screen where the patient performs the exercise.

- HELP

It displays a help message if patient requires so.

- BACK_HELP

This reconstructs the answer screen if it has been interrupted by a help call.

- RESULTS

Screen where results are numerically and graphically presented.

\section{B. Development}

Once the design of each task is accomplished, the development process begins. For this, Java programming language is used. The use of this language instead of $\mathrm{C}$ is due to several reasons: Java is open source, multiplatform and has a large number of libraries that offer many possibilities for development. Moreover, PREVIRNEC platform, on which tasks run, has been developed in this language, which means a restriction factor for selecting another programming language.

The programming scheme followed is based on a parent class, whose properties are inherited by all the classes that constitute a task. In addition, there is a set of classes that encapsulate a variety of common attributes and functions that are necessary for the development, representing entities such as the display, the counting of the user's score or the launching of the task itself.

Task loading process includes a series of input parameters (contained in a common XML file for all tasks) that enable its configuration. These parameters are different for each type of task and are intended to control the difficulty of the exercise, since they determine the number of stimuli, the time they are presented to the user, the time gap between stimuli and between screens, etc.

Stimuli can be of several types, from words, images, pictures or sounds to a combination of any of them in series or groups. A common directory contains common images (logos, buttons, etc.) and subdirectories with the specific images used as stimuli in each task. The sounds are stored with the same structure.

A second file sets the configuration of language, another input parameter that enables the choice of the language in which the task is performed (Spanish or Catalan). Texts displayed on screen (instructions, help messages, etc.) are stored in text files in different directories, one for each available language. Depending on the chosen language, the task will load the texts from one directory or another.

Once a task is completed, a set of output parameters are generated. These data, which summarize the user's activity during the exercise, are saved in an XML file (also common to all tasks) and displayed on the RESULTS screen. These results are also sent to the server to be stored in a database.

\section{REsULtS}

As a result of the design and development process described above, a set of 2D cognitive rehabilitation tasks has been generated.

Tasks consist of many different activities that make it possible to exercise a cognitive function through different methods: remembering or recognizing series of pictures or numbers (see Fig. 3), sequencing activities of daily living, grouping elements, deducting logical series, etc.

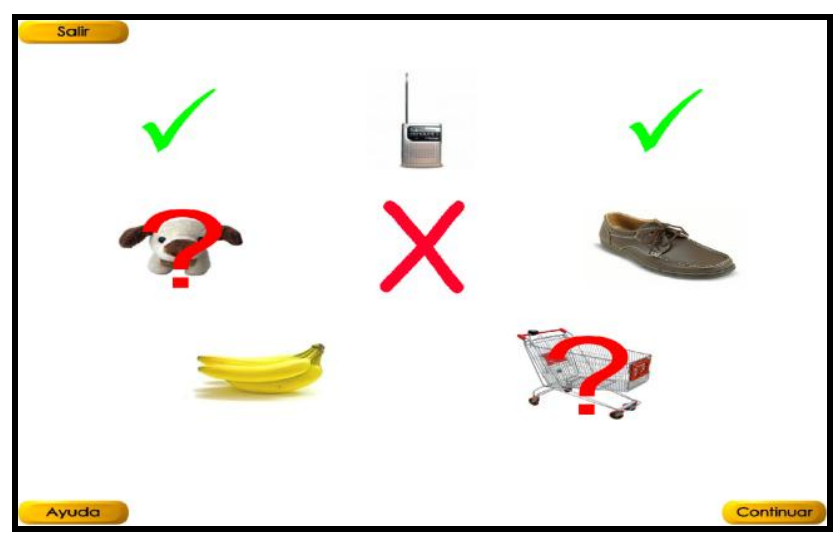

Fig 3. Example of a memory task

Results obtained by the patient in each session are stored in a database, so they can be analyzed asynchronously. Regarding the patient, RESULTS SCREEN provides feedback on the performance of the task, as shown in Fig. 4.

Analysis of the result data determine whether the patient is within the thresholds that define the so-called 'therapeutic range' (the one in which the execution of the tasks is clinically useful for the patient) or not. Consequently, task parameters will be reconfigured for the next session. Thus, the evolution of the patient determines the course of his therapy, adjusting automatically to achieve an improvement in the rehabilitation process.

Once a technological evaluation has been carried out, tasks are incorporated into the PREVIRNEC platform [9]. Today, this program has been followed by a total of 290 patients from different clinical centers in Spain.

From the results extracted from the sessions by a sample of patients, most of them from the Institut Guttmann, a clinical evaluation has been conducted. These patients were subjected to a set of neuropsychological test before and after PREVIRNEC programme to check if they had improved their cognitive profile. 


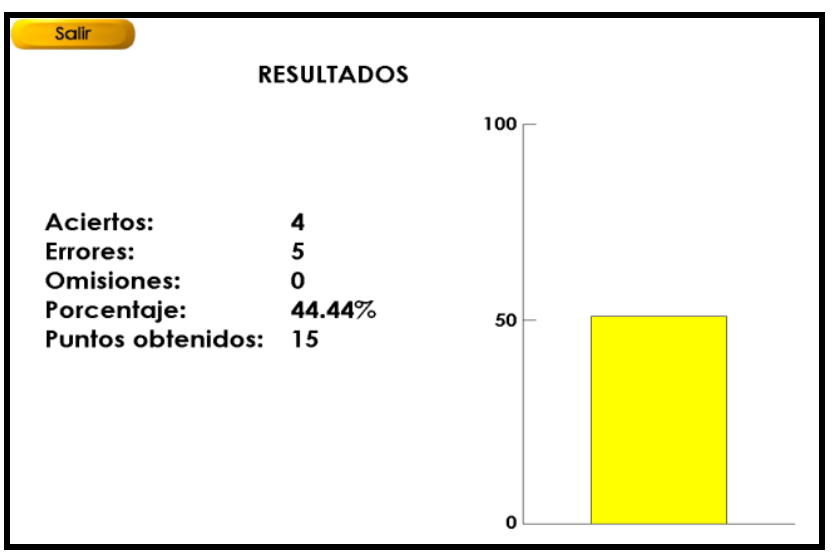

Fig. 4 Results screen

From a sample of 123 patients, 111 (90.24\%) had experienced a global improvement, i.e. in a cognitive function (attention, memory or executive functions), without affecting the rest, while 8 patients $(6.5 \%)$ had not changed significantly, and only 4 of them $(3.25 \%)$ had worsened their cognitive impairment profile.

\section{DisCUSSION}

The abovementioned clinical trial demonstrates the feasibility of the implemented tasks and its potential clinical impact in a significant improvement of cognitive functions in patients with acquired brain injury.

One advantage of this work is that the system configuration and data collection of the tasks makes no necessary the physical presence of the therapist during the execution, enabling the patient to perform the treatment outside the rehabilitation center. Therapist does not need to supervise each session of a patient, but to design and analyze the results obtained therein. Thus, therapist's workload decreases and the number of patients that can be attended by one therapist increases.

This work also allows personalization and individualization of therapies, and this is the main advantage comparing to the traditional therapy.

\section{Conclusions}

In this paper we have presented the design and development phases of new cognitive rehabilitation tasks based on ICTs in Biomedical Engineering, which offer new approaches to neuropsychological rehabilitation of memory, attention and executive functions in patients with acquired brain injury.

As we can draw from the results, these tasks improve and optimize the rehabilitation service in the great majority of the 290 patients (more than 90\%) that has followed the PREVIRNEC programme up to date, providing them a personalized environment of pleasant and comfortable rehabilitation that improves both their quality of life and their family environment during the therapy.

\section{AcKnowledgment}

The clinical evaluation has been conducted within the FIS ETES "Implantación clínica de PREVIRNEC en pacientes con alteraciones cognitivas de ICTUS y TCE" project (PI08/90525).

This research has been partially supported by the Avanza NEUROLEARNING project (TSI-020501-2008).

\section{REFERENCES}

1. World Health Organization. (2006) Neurological Disorders: Public health challenge, pp 30-37 ISBN 9241563362

2. Bernabeu M, Roig T (2002) Unidad de daño cerebral en el Instituto Guttmann. Hospital de neurorrehabilitación. Minusval 'Especial Rehabilitación del Daño Cerebral': 76-81 ISSN: 0210-0622

3. Valdizán JR (2008) Funciones cognitivas y redes neuronales del cerebro social. Rev Neurol supl 1: 65-68

4. Leh SE (2009) The Neural Circuitry of Executive Functions in Healthy Subjects and Parkinson's Disease. Neuropsychopharmacology Reviews (2009): 1-16

5. Papazian O, Alfonso I, Luzondo RJ (2006) Transtornos de las funciones ejecutivas. Rev Neurol supl 3: 45-50

6. De Noreña D et al. (2010) Effectiveness of neuropsychological rehabilitation in acquired brain injury (II): Executive functions, behavioural modification and psychotherapy, and the use of the new technologies. Rev Neurol. 2010 Dec 16;51(12):733-44

7. Tormos JM et al (2009) Information and communication technology in learning development and rehabilitation. International Journal of Integrated Care, ISSN 1568-4156

8. Égerházi A et al. Automated Neuropsychological Test Battery (CANTAB) in mild cognitive impairment and in Alzheimer's disease. Progress in Neuro-Psychopharmacology \& Biological Psychiatry 31 (2007) 746-751

9. García Molina A et al (2010) Clinical Program of Cognitive TeleRehabilitation for Traumatic Brain Injury. eChallenges e-2010 Conference, ISBN: 978-1-905824-21-2

\section{Author: Ruth Caballero Hernández}

Institute:Grupo de Bioingeniería y telemedicina (UPM)

Street: Avenida Complutense $\mathrm{n}^{\circ} 30$

City: Madrid

Country: Spain

Email: rcaballero@gbt.tfo.upm.es 